\title{
Complement C3 and Nonalcoholic Fatty Liver Disease in Chronic Kidney Disease Patients: A Pilot Study
}

\author{
Binbin Pan ${ }^{a} \quad$ Xin Wan ${ }^{a}$ Mengqing Ma ${ }^{b}$ Changchun Cao ${ }^{b}$ \\ a Department of Nephrology, Nanjing First Hospital, Nanjing Medical University, Nanjing, \\ PR China; ${ }^{b}$ Department of Nephrology, Sir Run Run Hospital, Nanjing Medical University, \\ Nanjing, PR China
}

\section{Keywords}

Nonalcoholic fatty liver disease $\cdot$ Chronic kidney disease $\cdot$ Complement C3

\begin{abstract}
Context: Evidences have suggested complement C3 is a biomarker for nonalcoholic fatty liver disease (NAFLD) in the general population. Objective: The present study was conducted to explore the predictive function of C3 for NAFLD in chronic kidney disease (CKD) patients. Design, Setting, and Participants: CKD patients were recruited for evaluation of their liver function, kidney function, serum lipids, glycated hemoglobin, blood, and immune function. The glomerular filtration rate was calculated using the CKD-EPI equation. NAFLD was diagnosed according to predefined ultrasonographic criteria. Results: A total of 648 consecutive CKD patients were included, with 216 (33.3\%) patients diagnosed with NAFLD. The NAFLD group had significant higher levels of serum protein, serum albumin, triglycerides, glycated hemoglobin, complement $C 3$, hemoglobin $(p=0.001)$, alanine aminotransferase $(p=0.002)$, estimated glomerular filtration rate $(p=0.007)$, and C4 $(p=0.043)$ and lower levels of cystatin $C, \beta_{2}$-microglobulin, proteinuria $(p=0.001)$, and high-density lipoprotein cholesterol $(p=$ 0.008). In a logistic regression model, only complement C3 (OR $=1.003 ; 95 \% \mathrm{Cl} 1.002-1.004$, $p=0.001$ ) was associated with a higher likelihood of being diagnosed with NAFLD. Finally, we constructed ROC curves for complement C3 for prediction of having NAFLD. The best cut-off for complement C3 was $993.5 \mathrm{mg} / \mathrm{L}$ and it yielded a sensitivity of $63.9 \%$ and a specificity of $70.1 \%$. Conclusion: Our study revealed that complement C3 can be used as a surrogate biomarker of NAFLD in CKD patients.




\section{Kidney \\ Blood Pressure \\ Research}

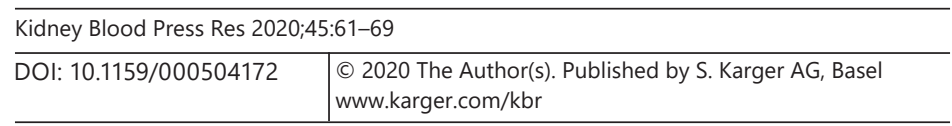

Pan et al.: Complement C3 and NAFLD

\section{Introduction}

Chronic kidney disease (CKD) is a common disorder all over the world, with a prevalence of more than $10 \%$ in China [1]. A numbers of diseases contribute to the development of CKD, including chronic nephritis, diabetes mellitus, hypertension, lupus, and thyroidal dysfunction [2]. Lipid accumulation, another common metabolic disorder, may also be involved in kidney injury. The injury is named lipid nephrotoxicity and was first outlined by Moorhead et al. [3], who proposed that dyslipidemia might contribute to the progression of renal disease in 1982. However, no data to date are available about the absolute correlation between dyslipidemia and CKD.

Meanwhile, dyslipidemia in the liver induces nonalcoholic fatty liver disease (NAFLD) which incorporates a spectrum of conditions ranging from simple steatosis to steatohepatitis, advanced fibrosis, and cirrhosis. NAFLD is emerging as the most common cause of chronic liver disease worldwide [4]. In addition, NAFLD has been proven to be a strong independent risk factor for cardiovascular events ( $\mathrm{HR}=2.03$; 95\% CI 1.33-3.13; $p<0.01$ ), but it was not associated with all-cause mortality (HR $=0.79 ; 95 \%$ CI $0.58-1.08 ; p=0.14$ ) or CKD progression ( $p=0.09$ for rate of decline of the estimated glomerular filtration rate [eGFR] slope) [5]. However, a number of studies have revealed the predictive function of NAFLD for CKD [6-10].

Several proinflammatory and oxidative stress mechanisms have been postulated to explain the relationship between these 2 conditions $[11,12]$. Insulin resistance has also been suggested to contribute to the progression of kidney disease by worsening renal hemodynamics through activation of the sympathetic nervous system, sodium retention, and downregulation of the natriuretic peptide system, making IR a possible mechanistic link between NAFLD and CKD [10]. Activated complement has also been indicated to be involved in NAFLD $[13,14]$ and CKD $[15,16]$. NAFLD patients showed hepatic deposition of activated C3 and C4d. C1q, as well as mannose-binding lectin accumulation, was found in most activated C3-positive patients. Strikingly, 50\% of the activated C3-positive patients also displayed membrane attack complex-associated C9 deposition. Deposition of complement factors was predominantly seen around hepatocytes with macrovesicularsteatosis [17].

In the light of this background, this study aimed to explore the possible role of complement C3 as a candidate biomarker for detection of NAFLD in CKD patients.

\section{Materials and Methods}

\section{Patients}

A total of 648 nondialysis CKD patients, including 428 hypertensive patients and 220 nonhypertensive patients, from August 2009 to October 2012 were registered via the case record system. We excluded subjects who were younger than 18 years, women who were pregnant, those who had a current or past history of significant alcohol abuse ( $\geq 30 \mathrm{~g} /$ day in men and $\geq 20 \mathrm{~g}$ /day in women), a previous diagnosis of HBV- or HCV-related liver disease, a primary or metastatic malignancy of the liver, Wilson's disease, an $\alpha_{1}$-antitrypsin deficiency, genetic hemochromatosis or other rare causes of liver dysfunction, autoimmune liver diseases, or parenteral nutrition, and those who were taking drugs potentially causing NAFLD, except for those used in treatment of CKD.

\section{Laboratory Measures}

Serum creatinine (Scr), cystatin $\mathrm{C}$, uric acid, $\beta_{2}$-microglobin, alanine aminotransferase (ALT), aspartate aminotransferase (AST), serum protein, albumin, and proteinuria were 


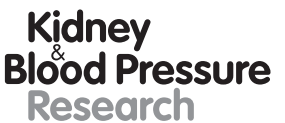

Kidney
Blood Pressure
Research \begin{tabular}{l|l}
\hline Kidney Blood Press Res 2020:45:61-69 \\
\hline DOI: 10.1159/000504172 & $\begin{array}{l}\text { @ 2020 The Author(s). Published by S. Karger AG, Basel } \\
\text { www.karger.com/kbr }\end{array}$ \\
\hline
\end{tabular}

Pan et al.: Complement C3 and NAFLD

measured using an OLYMPUS AU5400 automatic biochemical analyzer (Olympus Corporation, Mishima, Japan). The calibrators for the enzymatic method were traceable to an isotope dilution mass spectrometric method for Scr using standard reference methods (NIST SRM 967) [18]. Hemoglobin (Hb) was detected by the Sysmex XT-1800i Automated Hematology System (Shanghai, China). The concentrations of serum C-reactive protein were measured using electrochemiluminescence by ELECSYS 2010 (Roche, Switzerland). Glycated $\mathrm{Hb}(\mathrm{HbA1c})$ was determined using a BioRad VARIANT II HPLC analyzer (Biorad, Hemel Hempstead, UK). The values of serum immunoglobulin and complement were measured by singe immunodiffusion.

\section{Definition of CKD}

CKD stages were defined in accordance with KIDGO guidelines as follows: stage 1: eGFR $\geq 90 \mathrm{~mL} / \mathrm{min} / 1.73 \mathrm{~m}^{2}$, stage $2: 60 \mathrm{~mL} / \mathrm{min} / 1.73 \mathrm{~m}^{2} \leq \mathrm{eGFR}<90 \mathrm{~mL} / \mathrm{min} / 1.73 \mathrm{~m}^{2}$, stage $3: 30$ $\mathrm{mL} / \mathrm{min} / 1.73 \mathrm{~m}^{2} \leq \mathrm{eGFR}<60 \mathrm{~mL} / \mathrm{min} / 1.73 \mathrm{~m}^{2}$, stage $4: 15 \mathrm{~mL} / \mathrm{min} / 1.73 \mathrm{~m}^{2} \leq \mathrm{eGFR}<30 \mathrm{~mL} /$ $\mathrm{min} / 1.73 \mathrm{~m}^{2}$, and stage 5 : eGFR $<15 \mathrm{~mL} / \mathrm{min} / 1.73 \mathrm{~m}^{2}$.

eGFR was calculated using the Chronic Kidney Disease Epidemiology Collaboration (CKD-EPI) 4-level race equation [19, 20] and the last Scr value before discharge was selected as the baseline level. The specific CKD-EPI 4-level race GFR estimation equation is shown in Table 1.

The expressions of the eGFR equation were as follows:

$$
\begin{aligned}
& \mathrm{eGFR}=\operatorname{EXP}(\operatorname{LN}[51]-0.328 \times \operatorname{LN}[\mathrm{Scr} / 88.4 / 0.7]+\text { age } \times \operatorname{LN}[0.993]) \text { (if female and creatinine }<0.7) ; \\
& \mathrm{eGFR}=\operatorname{EXP}(\operatorname{LN}[151]-1.210 \times \mathrm{LN}[\mathrm{Scr} / 88.4 / 0.7]+\text { age } \times \operatorname{LN}[0.993]) \text { (if female and creatinine } \geq 0.7) ; \\
& \mathrm{eGFR}=\operatorname{EXP}(\operatorname{LN}[149]-0.412 \times \operatorname{LN}[\mathrm{Scr} / 88.4 / 0.9]+\text { age } \times \operatorname{LN}[0.993]) \text { (if male and creatinine }<0.9) ; \\
& \mathrm{eGFR}=\operatorname{EXP}(\operatorname{LN}[149]-1.210 \times \operatorname{LN}[\mathrm{Scr} / 88.4 / 0.9]+\text { age } \times \operatorname{LN}[0.993])(\text { if male and creatinine } \geq 0.9)
\end{aligned}
$$

\section{Definition of NAFLD}

NAFLD was diagnosed based on the presence of a characteristic ultrasound (US) pattern, including diffuse hyperechogenicity of the liver parenchyma compared to the kidney cortex, deep beam attenuation, and poor visualization of the intrahepatic vessels and the diaphragm border [21]. Liver ultrasonography was performed after overnight fasting by an experienced sonographer blinded to patients' clinical and laboratory data using a 3.5. to 5-MHz convex probe. The study image was then analyzed by another sonographer who was also blinded to the patients' clinical and laboratory data, and the final assignment to an US category (NAFLD yes/no) was done by agreement of the 2 operators.

\section{Statistical Analysis}

Statistics analysis was performed using PASW 18.0 statistical software (SPSS Inc., Chicago, IL, USA). Data were expressed as means \pm SD. The prevalence of NAFLD in different CKD stages was calculated using the $\chi^{2}$ test. Student's $t$ test was used to compare means for continuous variables. Fisher's exact test was used to compare prevalence dichotomic variables. Binary logistic regression was employed to explore risk factors for NAFLD. Multicolinearity between independent variables was evaluated by using the variance inflation factor before entering each value in the regression model. $p<0.05$ was considered statistically significant. Receiver operating characteristic (ROC) curves were built to evaluate the predictive value of significant variables for the likelihood of having NAFLD. The best cut-off for ROC curves was calculated with the Youden index. 
Table 1. Characteristics of the subjects in the CKD and CKD-NAFLD groups

\begin{tabular}{|c|c|c|c|}
\hline & $\begin{array}{l}\text { CKD } \\
(n=432)\end{array}$ & $\begin{array}{l}\text { CKD-NAFLA } \\
(n=216)\end{array}$ & $p$ value \\
\hline Age, years & $65.15 \pm 17.48$ & $64.92 \pm 14.74$ & 0.86 \\
\hline Male sex & 212 (49.1) & $94(43.5)$ & 0.211 \\
\hline ALT, UI/L & $20.78 \pm 22.54$ & $26.78 \pm 23.9$ & $0.002^{\#}$ \\
\hline AST, UI/L & $25.91 \pm 21.84$ & $25.72 \pm 13.44$ & 0.588 \\
\hline Serum protein, g/dL & $59.88 \pm 9.63$ & $64.92 \pm 8.28$ & $0.000^{\#}$ \\
\hline Serum albumin, g/dL & $34.64 \pm 6.31$ & $37.98 \pm 5.33$ & $0.000^{\#}$ \\
\hline Glutamyltransferase, g/dL & $35.65 \pm 54.48$ & $43.95 \pm 66.72$ & 0.091 \\
\hline Cystatin C, mg/dL & $2.18 \pm 1.37$ & $1.65 \pm 0.8$ & $0.000^{\#}$ \\
\hline$\beta_{2}$-microglobulin, mg/dL & $5.81 \pm 4.28$ & $4.04 \pm 3.8$ & $0.000^{\#}$ \\
\hline Urine acid, $\mu \mathrm{mol} / \mathrm{L}$ & $403.26 \pm 157.95$ & $399.45 \pm 133.21$ & 0.747 \\
\hline $\mathrm{eGFR}, \mathrm{mL} / \mathrm{min} \times 1.73 \mathrm{~m}^{2}$ & $57.92 \pm 38.54$ & $66.11 \pm 29.7$ & $0.007^{\#}$ \\
\hline Total cholesterol, mmol/L & $4.65 \pm 1.76$ & $4.79 \pm 1.47$ & 0.293 \\
\hline Triglycerides, mmol/l & $1.49 \pm 0.99$ & $2.12 \pm 1.47$ & $0.000^{\#}$ \\
\hline HDL cholesterol, mmol/L & $1.11 \pm 0.35$ & $1.04 \pm 0.28$ & $0.008^{\#}$ \\
\hline LDL cholesterol, mmol/L & $2.86 \pm 1.29$ & $2.93 \pm 1.12$ & 0.481 \\
\hline Glycated hemoglobin & $6.04 \pm 1.27$ & $6.48 \pm 1.24$ & $0.000^{\#}$ \\
\hline $\mathrm{IgG}, \mathrm{mg} / \mathrm{L}$ & $11.41 \pm 5.85$ & $11.96 \pm 3.47$ & 0.2 \\
\hline $\mathrm{IgM}, \mathrm{mg} / \mathrm{L}$ & $1.03 \pm 1.13$ & $0.91 \pm 0.49$ & 0.128 \\
\hline $\operatorname{IgA}, \mathrm{mg} / \mathrm{L}$ & $2.40 \pm 1.45$ & $2.51 \pm 1.1$ & 0.324 \\
\hline $\mathrm{C} 3, \mathrm{mg} / \mathrm{L}$ & $890.5 \pm 239.92$ & $1,085.3 \pm 270$ & $0.000^{\#}$ \\
\hline $\mathrm{C} 4, \mathrm{mg} / \mathrm{L}$ & $218.65 \pm 69.9$ & $230.39 \pm 68.98$ & $0.043^{*}$ \\
\hline $\mathrm{CRP}, \mathrm{mg} / \mathrm{L}$ & $15.97 \pm 33.03$ & $22.54 \pm 54.6$ & 0.115 \\
\hline T2DM & 135 (31.3) & $93(43.1)$ & $0.003^{\#}$ \\
\hline Proteinuria, g/24 h & $1.27 \pm 2.11$ & $0.77 \pm 1.60$ & $0.000^{\#}$ \\
\hline $\mathrm{Hb}, \mathrm{g} / \mathrm{L}$ & $117.83 \pm 21.62$ & $129.1 \pm 17.28$ & $0.000^{\#}$ \\
\hline Hypertension, $n(\%)$ & $270(62.5)$ & $158(73.1)$ & $0.007^{\#}$ \\
\hline Albuminuria $(n=456)$ & $243 / 286$ & $141 / 170$ & $0.01^{*}$ \\
\hline
\end{tabular}

Values are presented as means \pm SD or numbers (\%). HDL, high-density lipoprotein; LDL, low-density lipoprotein; IgG, immunoglobulin G; IgM, immunoglobulin M; IgA, immunoglobulin A; CRP, C-reactive protein; T2DM, type 2 diabetes mellitus. ${ }^{*} p<0.05$. ${ }^{*} p<0.01$.

\section{Results}

\section{Characteristics of Subjects in the CKD and CKD-NAFLD Groups}

A total of 648 consecutive CKD patients were recruited and 216 (33.3\%) patients were diagnosed with NAFLD. The causes of CKD were chronic glomerulonephritis, asymptomatic hematuria, diabetic nephropathy, polycystic kidney disease, chronic renal failure (of unknown or indeterminate etiology), hypertensive nephropathy, and obstructive nephropathy. The NAFLD group had significant higher levels of serum protein, serum albumin, triglycerides, HbA1c, complement C3, Hb $(p=0.001)$, ALT $(p=0.002)$, eGFR $(p=0.007)$, and C4 $(p=0.043)$ and a higher frequency of hypertension $(p=0.007)$. Additionally, markedly lower levels of cystatin $C, \beta_{2}$-microglobulin, proteinuria $(p=0.001)$, and high-density lipoprotein cholesterol ( $p=0.008$ ) and a lower frequency of albuminuria (only a total of 72 patients had albuminuria values within the normal range, while 384 patients had values exceeding the normal range) were also observed in the NAFLD group.

The prevalence of NAFLD in each CKD group is shown in Table 2, with a significantly decreasing trend from CKD stage 1 to CKD stage 5. Additionally, levels of serum protein and albumin revealed a declining trend with increasing CKD stages (Tables 1, 2). 
Kidney

Blood Pressure

Research

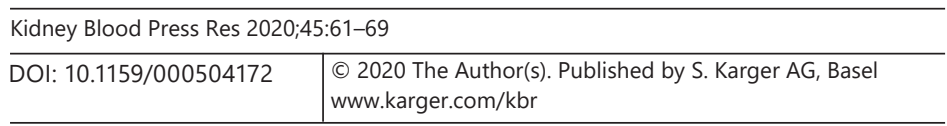

Pan et al.: Complement C3 and NAFLD
Table 2. Prevalence of NAFLD in the different CKD stages

\begin{tabular}{lclll}
\hline & $\begin{array}{l}\text { Non-NAFLD } \\
(n=432), n\end{array}$ & $\begin{array}{l}\text { NAFLD } \\
(n=216), n(\%)\end{array}$ & $\begin{array}{l}\text { Albumin, } \\
\mathrm{g} / \mathrm{L}\end{array}$ & $\begin{array}{l}\text { Protein, } \\
\mathrm{g} / \mathrm{L}\end{array}$ \\
\hline CKD1 & 112 & $73(33.8)$ & 37.12 & 62.64 \\
CKD2 & 66 & $48(22.2)$ & 36.3 & 62.66 \\
CKD3 & 63 & $42(19.4)$ & 36 & 61.64 \\
CKD4 & 140 & $50(23.1)$ & 36.15 & 62.62 \\
CKD5 & 51 & $3(1.4)$ & 32.44 & 57.7 \\
\hline
\end{tabular}

\begin{tabular}{llll}
\hline & OR & $95 \%$ CI & $p$ value \\
\hline Male sex & 1.069 & $0.704-1.623$ & 0.753 \\
C3 & 1.003 & $1.002-1.004$ & $0.0001^{\#}$ \\
Age & 1.011 & $0.995-1.028$ & 0.182 \\
CRP & 1.001 & $0.996-1.005$ & 0.691 \\
Glycated Hb & 1.164 & $0.993-1.365$ & 0.061 \\
eGFR & 1.006 & $0.999-1.013$ & 0.113 \\
\hline
\end{tabular}

CRP, C-reactive protein. ${ }^{*} p<0.01$.
Table 3. Logistic regression analysis using NAFLD as the dependent variable 
Fig. 1. ROC curves for complement C3 for prediction of NAFLD. The area under the ROC was 0.722 (95\% CI 0.681-0.764, $p<0.001$ ) for C3. The analysis of ROC revealed that the optimal cut-off for complement C3 was $993.5 \mathrm{mg} / \mathrm{L}$ and provided a sensitivity of $63.9 \%$ and a specificity of $70.1 \%$.

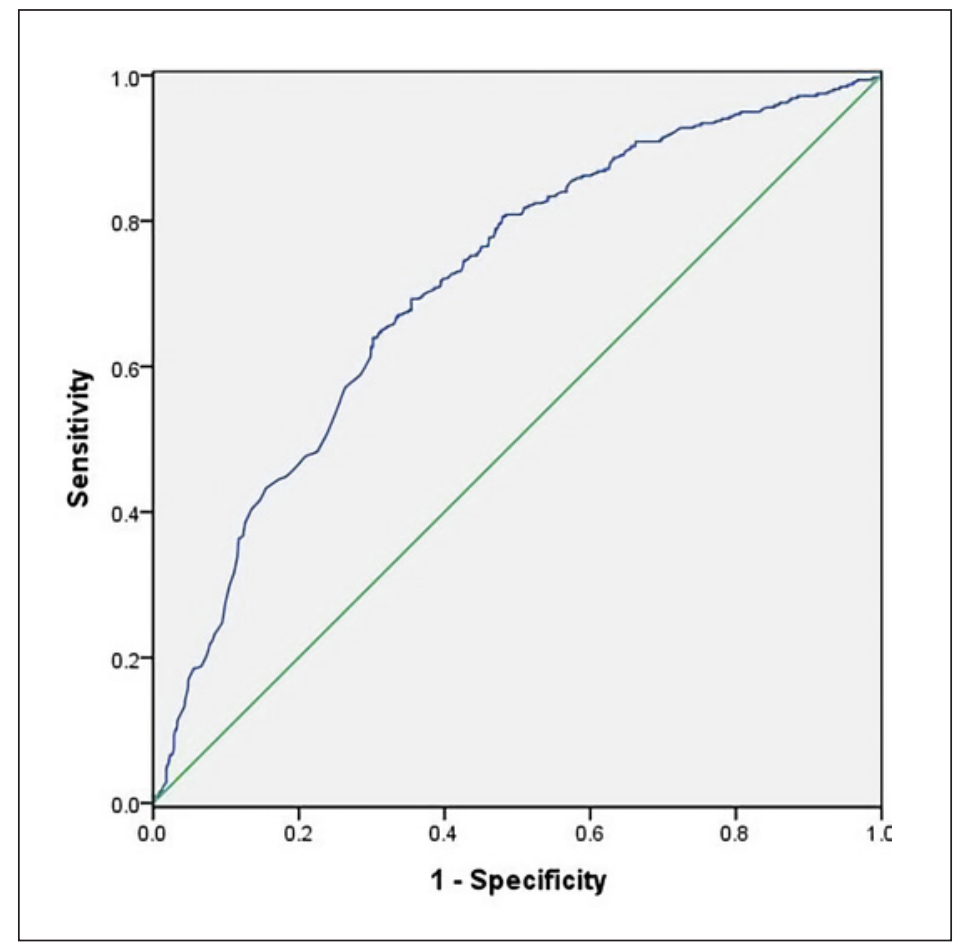

to more CKD stage 1 to CKD stage 3 patients in the NAFLD group (241/432 vs. 163/216). However, complement C3 and C4 were both elevated in NAFLD and the logistic regression showed that C3 was an independent risk factor for NAFLD, indicating that complement system may be involved in the development of NAFLD. Furthermore, C3 had a promising prediction efficiency for NAFLD, with a sensitivity of $63.9 \%$ and a specificity of $70.1 \%$.

Emerging amounts of epidemiologic data have shown an association between the prevalence and severity of NAFLD and the incidence and stage of CKD. A cohort study showed that NAFLD proved to be a strong independent risk factor for cardiovascular events $(\mathrm{HR}=2.03$; $95 \%$ CI 1.33-3.13; $p<0.01$ ), but it was not associated with all-cause mortality (HR $=0.79$; 95\% CI 0.58-1.08; $p=0.14$ ) or CKD progression [5]. Nevertheless, a total of 9 observational studies with 96,595 adult individuals (34.1\% with NAFLD) of predominantly Asian descent and 4,653 cases of incident CKD stage $\geq 3$ (i.e., defined as the occurrence of an eGFR $<60 \mathrm{~mL}$ / $\min / 1.73 \mathrm{~m}^{2}$, with or without accompanying overt proteinuria) over a median period of 5.2 years showed that NAFLD (detected by biochemistry, the fatty liver index, or ultrasonography) is associated with a nearly $40 \%$ increase in the long-term risk of incident CKD [22]. Furthermore, a retrospective cohort study of 41,430 adult men and women (average age, 48.9 years) without CKD at baseline NAFLD was associated with an increased risk of CKD development. NAFLD may adversely affect renal function and patients may need to be carefully monitored for an increased risk of CKD [6].

When it comes to the pathogenesis of NAFLD, IR is the key pathophysiological factor for the development and progression of NAFLD. In addition, IR has been attributed to the development of CKD [23]. Other pathophysiological mechanisms, such as fetuin-A, adiponectin [24], and angiotensin II may be contributing factors for CKD development in patients with NAFLD [11]. The complement system may also be associated with the development of CKD and NAFLD. C3 is mainly synthesized in hepatocytes and undetectable in the liver of healthy subjects. However, $74 \%$ of the NAFLD patients showed hepatic deposition of activated C3 and 


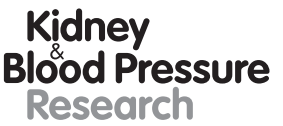

Research

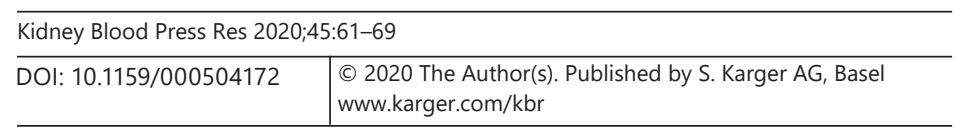

Pan et al.: Complement C3 and NAFLD

C4d [17]. C3 levels were positively associated with both NAFLD prevalence and severity regardless of whether metabolic syndromes exist or not [13]. Little data is available about whether C3 is directly a pathogen of NAFLD or not; however, C3 may induce the progression of NAFLD [17]. Moreover, C3 is an important immune regulatory factor in the pathogenesis of CKD [25-30]. Complement systems including C3 have been involved in most of cases of nephropathy, e.g., membrane nephropathy, lupus nephropathy, IgA nephropathy, and even diabetic nephropathy. In our study, C3 was an independent influencing factor for NAFLD, which is similar to the results of the previous study $[13,14]$. We speculate that inflammation in CKD patients induces activation of the complement system which may result in deposition of activated $\mathrm{C} 3$ in the liver to assemble membrane attack complex (MAC) [17]. After deposition of $\mathrm{C} 3$ in the liver, activating $\mathrm{C} 5$ and formation of MAC emerge, which induces liver injury. However, to date, no data are available regarding the prediction efficiency of $\mathrm{C} 3$ for NAFLD in CKD. A study conducted in rheumatoid arthritis patients showed that the best cut-off of complement C3 for predicting NAFLD was $1.23 \mathrm{~g} / \mathrm{L}$ and yielded a sensitivity of $76 \%$ and a specificity of $64 \%$ [14]. Our study also revealed sensitivity and specificity values comparable to the above result, suggesting that $\mathrm{C} 3$ can be used as a potential marker for predicting NAFLD in CKD.

There are 2 limitations to our study. Firstly, the BMI was not obtained. As we all know, the BMI is an important index to assess metabolic syndrome. It is associated with NAFLD, obesity, and diabetes. In our study, levels of serum lipids and HbA1c in CKD-NAFLD were both higher than those in CKD without NAFLD, suggesting that an abnormal metabolic status exists in these patients although the BMI was not recorded.

Secondly, histological confirmation of the diagnosis of NAFLD was deficient. However, patients in our cohort manifested without signs or symptoms of clinically relevant liver disease, including normal or near normal liver function indices, with only a small fraction of participants exhibiting an increase in ALT levels exceeding the upper limit of normal. Therefore, a liver biopsy may induce unbalanced ethical issues.

Taken together, C3 in CKD patients not only represent activity of kidney disease but it can also be used as a candidate biomarker for NAFLD.

\section{Statement of Ethics}

This study was performed in accordance with the Declaration of Helsinki and was approved by the Institutional Review Board. Informed consent was obtained from all of the participants when they were admitted to the hospital.

\section{Disclosure Statement}

The authors have nothing to disclose.

\section{Funding Sources}

This work was supported by grants from the Foundation of Science and Technology Development Program, Nanjing Medical University (NMUB2018321), and the Natural Science Foundation of Jiangsu Province (SBK2017020756). The funders had no role in the study design, data collection and analysis, the decision to publish, or the preparation of this paper. 


\section{Kidney \\ Blood Pressure Research}

\begin{tabular}{l|l}
\hline Kidney Blood Press Res 2020;45:61-69 \\
\hline DOI: 10.1159/000504172 & $\begin{array}{l}\text { @ 2020 The Author(s). Published by S. Karger AG, Basel } \\
\text { www.karger.com/kbr }\end{array}$ \\
\hline
\end{tabular}

Pan et al.: Complement C3 and NAFLD

\section{Author Contributions}

The research was designed by B.P. and C.C. All of the authors helped to write this report and commented on it. B.P. and M.M. analyzed the data and advised on statistical issues at the time of the research write up. X.W. and C.C. were research administrators. M.M. was a research nurse responsible for recruitment and return of data.

\section{References}

1 Zhang L, Wang F, Wang L, Wang W, Liu B, Liu J, et al. Prevalence of chronic kidney disease in China: a crosssectional survey. Lancet. 2012 Mar;379(9818):815-22.

2 Du X, Pan B, Li W, Zou Y, Hua X, Huang W, et al. Albuminuria is an independent risk factor of T4 elevation in chronic kidney disease. Sci Rep. 2017 Jan; 7(1):41302.

3 Moorhead JF, Chan MK, El-Nahas M, Varghese Z. Lipid nephrotoxicity in chronic progressive glomerular and tubulo-interstitial disease. Lancet. 1982 Dec;2(8311):1309-11.

4 Vernon G, Baranova A, Younossi ZM. Systematic review: the epidemiology and natural history of non-alcoholic fatty liver disease and non-alcoholic steatohepatitis in adults. Aliment Pharmacol Ther. 2011 Aug;34(3):27485.

5 Chinnadurai R, Ritchie J, Green D, Kalra PA. Non-alcoholic fatty liver disease and clinical outcomes in chronic kidney disease. Nephrol Dial Transplant. 2019 Mar;34(3):449-57.

6 Sinn DH, Kang D, Jang HR, Gu S, Cho SJ, Paik SW, et al. Development of chronic kidney disease in patients with non-alcoholic fatty liver disease: A cohort study. J Hepatol. 2017 Dec;67(6):1274-80.

7 Machado MV, Gonçalves S, Carepa F, Coutinho J, Costa A, Cortez-Pinto H. Impaired renal function in morbid obese patients with nonalcoholic fatty liver disease. Liver Int. 2012 Feb;32(2):241-8.

8 Targher G, Mantovani A, Pichiri I, Mingolla L, Cavalieri V, Mantovani W, et al. Nonalcoholic fatty liver disease is independently associated with an increased incidence of chronic kidney disease in patients with type 1 diabetes. Diabetes Care. 2014 Jun;37(6):1729-36.

9 Targher G, Bertolini L, Rodella S, Zoppini G, Lippi G, Day C, et al. Non-alcoholic fatty liver disease is independently associated with an increased prevalence of chronic kidney disease and proliferative/laser-treated retinopathy in type 2 diabetic patients. Diabetologia. 2008 Mar;51(3):444-50.

10 Targher G, Byrne CD. Non-alcoholic fatty liver disease: an emerging driving force in chronic kidney disease. Nat Rev Nephrol. 2017 May;13(5):297-310.

11 Marcuccilli M, Chonchol M. NAFLD and chronic kidney disease. Int J Mol Sci 2016;17:562.

12 Musso G, Cassader M, Cohney S, Pinach S, Saba F, Gambino R. Emerging liver-kidney interactions in nonalcoholic fatty liver disease. Trends Mol Med. 2015 Oct;21(10):645-62.

13 Xu C, Chen Y, Xu L, Miao M, Li Y, Yu C. Serum complement C3 levels are associated with nonalcoholic fatty liver disease independently of metabolic features in Chinese population. Sci Rep. 2016 Mar;6(1):23279.

14 Ursini F, Russo E, Mauro D, Abenavoli L, Ammerata G, Serrao A, et al. Complement C3 and fatty liver disease in Rheumatoid arthritis patients: a cross-sectional study. Eur J Clin Invest. 2017 Oct;47(10):728-35.

15 Ravindran A, Fervenza FC, Smith RJ, Sethi S. C3 glomerulopathy associated with monoclonal Ig is a distinct subtype. Kidney Int. 2018 Jul;94(1):178-86.

16 Li QY, Li HY, Fu G, Yu F, Wu Y, Zhao MH. Autoantibodies against C-Reactive Protein Influence Complement Activation and Clinical Course in Lupus Nephritis. J Am Soc Nephrol. 2017 Oct;28(10):3044-54.

17 Rensen SS, Slaats Y, Driessen A, Peutz-Kootstra CJ, Nijhuis J, Steffensen R, et al. Activation of the complement system in human nonalcoholic fatty liver disease. Hepatology. 2009 Dec;50(6):1809-17.

18 Dodder NG, Tai SS, Sniegoski LT, Zhang NF, Welch MJ. Certification of creatinine in a human serum reference material by GC-MS and LC-MS. Clin Chem. 2007 Sep;53(9):1694-9.

19 Du X, Liu L, Hu B, Wang F, Wan X, Jiang L, et al. Is the Chronic Kidney Disease Epidemiology Collaboration fourlevel race equation better than the cystatin C equation? Nephrology (Carlton). 2012 May;17(4):407-14.

20 Stevens LA, Claybon MA, Schmid CH, Chen J, Horio M, Imai E, et al. Evaluation of the Chronic Kidney Disease Epidemiology Collaboration equation for estimating the glomerular filtration rate in multiple ethnicities. Kidney Int. 2011 Mar;79(5):555-62.

21 Hernaez R, Lazo M, Bonekamp S, Kamel I, Brancati FL, Guallar E, et al. Diagnostic accuracy and reliability of ultrasonography for the detection of fatty liver: a meta-analysis. Hepatology. 2011 Sep;54(3):1082-90.

22 Mantovani A, Zaza G, Byrne CD, Lonardo A, Zoppini G, Bonora E, et al. Nonalcoholic fatty liver disease increases risk of incident chronic kidney disease: a systematic review and meta-analysis. Metabolism. 2018 Feb;79: 64-76.

23 Mikolasevic I, Milic S, Turk Wensveen T, Grgic I, Jakopcic I, Stimac D, et al. Nonalcoholic fatty liver disease - A multisystem disease? World J Gastroenterol. 2016 Nov;22(43):9488-505.

24 Ix JH, Sharma K. Mechanisms linking obesity, chronic kidney disease, and fatty liver disease: the roles of fetuin-A, adiponectin, and AMPK. J Am Soc Nephrol. 2010 Mar;21(3):406-12. 
25 Bao X, Borné Y, Muhammad IF, Schulz CA, Persson M, Orho-Melander M, et al. Complement C3 and incident hospitalization due to chronic kidney disease: a population-based cohort study. BMC Nephrol. 2019 Feb; 20(1):61.

26 Frémeaux-Bacchi V, Sellier-Leclerc AL, Vieira-Martins P, Limou S, Kwon T, Lahoche A, et al. Complement Gene Variants and Shiga Toxin-Producing Escherichia coli-Associated Hemolytic Uremic Syndrome: Retrospective Genetic and Clinical Study. Clin J Am Soc Nephrol. 2019 Mar;14(3):364-77.

27 Cernoch M, Hruba P, Kollar M, Mrazova P, Stranavova L, Lodererova A, et al. Intrarenal complement system transcripts in chronic antibody-mediated rejection and recurrent IgA nephropathy in kidney transplantation. Front Immunol. 2018 Oct; 9:2310.

28 Bomback AS, Santoriello D, Avasare RS, Regunathan-Shenk R, Canetta PA, Ahn W, et al. C3 glomerulonephritis and dense deposit disease share a similar disease course in a large United States cohort of patients with C3 glomerulopathy. Kidney Int. 2018 Apr;93(4):977-85.

29 Jullien P, Laurent B, Claisse G, Masson I, Dinic M, Thibaudin D, et al. Deletion Variants of CFHR1 and CFHR3 Associate with Mesangial Immune Deposits but Not with Progression of IgA Nephropathy. J Am Soc Nephrol. 2018 Feb;29(2):661-9.

30 Zhang YM, Gu QH, Huang J, Qu Z, Wang X, Meng LQ, et al. Clinical Significance of IgM and C3 Glomerular Deposition in Primary Focal Segmental Glomerulosclerosis. Clin J Am Soc Nephrol. 2016 Sep;11(9):1582-9. 